\title{
Rauchende Patienten: einige Behandlungsempfehlungen
}

Jacques Cornuz

Prof. Dr. med., Chefarzt, Poliklinik des Universitätsspitals, Lausanne
Die meisten Raucherinnen und Raucher wollen eines Tages mit dem Rauchen aufhören. Dieser Artikel gibt einige Empfehlungen für die Behandlung rauchender Patienten und Patientinnen.

Eine Frage wird derzeit häufig gestellt: «Herr/ Frau Doktor, soll ich die elektronische Zigarette versuchen?» Sie können antworten: «Sicherlich!» Für Personen, die regelmässig rauchen, ist es besser, zur E-Zigarette zu wechseln. Sie müssen aber darauf hingewiesen werden, dass das rauchbedingte Krankheitsrisiko nur partiell verringert wird, wenn dieser Wechsel unvollständig bleibt, also beide Produkte konsumiert werden (E-Zigaretten und Tabak), da selbst ein geringer Tabakkonsum (2-5 Zigaretten/ Tag) das Risiko erhöht, an tabakbedingten Erkrankungen zu sterben. Es gibt keine Daten, die belegen, dass die E-Zigarette eine wirksame Hilfe ist, um mit dem Rauchen aufzuhören

\section{«Bei der Nikotinsubstitution besteht keine Gefahr der Über- dosierung.»}

Um mit rauchenden Patientinnen und Patienten auf einer Wellenlänge zu sein, kann es hilfreich sein, den «Genuss»-Aspekt anzusprechen. Oft sprechen Raucherinnen und Raucher, selbst wenn sie sehr motiviert sind aufzuhören, von einigen «Genuss»Zigaretten, besonders denen am Morgen und nach dem Essen. Im Motivationsgespräch sollte jedoch vermittelt werden, dass dieser «Genuss» vor allem ein Belohnungsgefühl (rewarding) ist, das durch den erneuten Tabakkonsum nach einer zeitweiligen Unterbrechung (nachts und beim Essen) entsteht. Hier sollte den Patientinnen und Patienten gesagt werden, dass sie künftig diesem «Genuss» entsagen und sich auf einen gewissen Verlust einstellen müssen.

Wenn ein Patient oder eine Patientin entschlossen ist, nicht mehr zu rauchen, sind bei der Behandlung folgende Punkte zu beachten:

Bei jeder Person, die regelmässig raucht (mehr als Prof. Dr. med. Jacques Cornuz Policlinique médicale universitaire Quartier UNIL-CHU Rue du Bugnon 44 CH-1011 Lausanne Tel. 0213140506 Fax 0213146099 dauer (Inhalator, Kaugummi) zu empfehlen. Bei der Nikotinsubstitution besteht keine Gefahr der Überdosierung. Vielmehr besteht das Risiko einer Unterdosierung und somit eines Rückfalls aufgrund einer unzureichenden Substitution. Auch Vareniclin ist eine empfohlene Therapieoption. Die Patientin oder der Patient muss ein Aufhördatum festlegen. Damit wird eine Verpflichtung sich selbst und dem Arzt gegenüber eingegangen. Innerhalb von 48 bis 72 Stunden nach dem Aufhördatum sollte ein erneuter (telefonischer) Kontakt erfolgen. Bei dieser Gelegenheit sollte die Behandlung angepasst und der Patient oder die Patientin beglückwünscht werden.

Gründe für einen Rückfall sind: 1. nicht ausreichend geklärter Zweifel in Bezug auf den Rauchstopp; 2. eine unzureichende Nikotinsubstitution; 3. Druck durch Gleichaltrige oder den Partner oder die Partnerin; 4. Gewichtszunahme; 5. Gefühlsbewältigung. Die Erfahrung zeigt, dass Männer häufig bei positiven Anlässen rückfällig werden, zum Beispiel auf einer Party oder bei einem Sieg ihres Sportklubs. Frauen hingegen neigen in persönlich schwierigen Situationen mit negativen Gefühlen (Streit, Beziehungsprobleme) zu einem Rückfall.

\section{Rauchstopp-Wettbewerb 2014}

Zum Welttag ohne Tabak am 31. Mai findet erneut der Rauchstopp-Wettbewerb statt, dieses Jahr vom 2. bis 30. Juni. Mit dieser Aktion unterstützt das Nationale Rauchstopp-Programm die ärztliche Beratung zum Rauchstopp.

Die Geldpreise von einmal 5000 und zehnmal 500 Franken motivieren viele Raucherinnen und Raucher, einen Aufhörversuch zu machen. Mit jedem Versuch steigt die Chance für den endgültigen Ausstieg aus der Nikotinabhängigkeit.

Eine Anmeldung ist möglich online auf www. at-schweiz.ch (Deutsch, Französisch, Italienisch, Englisch und Türkisch) oder schriftlich mit der Wettbewerbskarte (zusätzlich auch Albanisch, Portugiesisch, Serbisch/Kroatisch/Bosnisch und Spanisch). Die Anmeldekarte, ein Plakat und weitere Unterlagen können kostenlos bestellt werden auf www.at-schweiz.ch oder unter der Telefonnummer 0315991020.

Partnerorganisationen des Wettbewerbs sind die Verbindung der Schweizer Ärztinnen und Ärzte FMH, pharmaSuisse - Schweizerischer Apothekerverband, die Schweizerische Zahnärzte-Gesellschaft, der Schweizer Berufsverband der Pflegefachfrauen und Pflegefachmänner und Swiss Dental Hygienists. Der Wettbewerb wird durch den Tabakpräventionsfonds finanziert. 